\title{
Analysis of Molding Behavior of PET Bottle Assumed as Newtonian Fluid for Fusion of 3D Molding In-label
}

\author{
Seong-Gyu Cho and Joon-Seong Lee \\ Dept. of Mechanical Engineering, Graduate School, Kyonggi University \\ Dept. of Mechanical System Engineering, Kyonggi University \\ jslee1@kyonggi.ac.kr
}

\begin{abstract}
Approximately $90 \%$ of the PET bottles used in the vicinity are used in beverage containers. These PET bottles are produced by a stretch blow molding process. The stretch blow molding is a molding process inside an opaque mold. Therefore, the molding process can not be visually observed. This is a great difficulty in interpreting or analyzing the molding process. Since stretch blow molding proceeds in an invisible state, it is important to establish an appropriate analysis method. In this study, the SBM process is divided into several stages, and the final interpretation is performed by combining the appropriate assumptions. In addition, PET bottles are assumed to be general Newtonian fluids and will be analyzed. The results of the analysis using these methods will be compared with the actual products to confirm that the analysis method is appropriate.
\end{abstract}

Keywords: Blow, Molding, Newtonian fluid, PET bottle, Stretch

\section{Introduction}

Approximately $90 \%$ of the PET bottles are used in beverage containers. These PET bottles are produced by a SBM(Stretch blow molding) process. The SBM refers to a method in which the preform is heated in advance, and then the stretching and the blow molding are simultaneously performed to process the preform into a desired shape[1]. The SBM is a molding process inside an opaque mold. Therefore, the molding process can not be visually observed. This is a great difficulty in interpreting or analyzing the molding process. Since SBM proceeds in an invisible state, it is important to establish an appropriate analysis method. When the elongation rate of the preform is large in the SBM, it is very difficult to predict the final thickness distribution of the PET bottle. Since the previous studies have been made for pure research purposes, it is difficult to apply the results directly to the field[2]. In the field, they use the method of modifying the molding process by looking at the defective product after producing the product. This method generates a large number of trial errors. Therefore, this method leads to an increase in the research cost[3]. The purpose of this study is to demonstrate a method that can save time and expense when using a commercial analysis program in the field of stretch blow molding analysis of PET bottles.

The physical properties of the preforms used in the field are given by density and intrinsic viscosity. However, intrinsic viscosity is a numerical value for obtaining the molecular weight of a polymer compound, so it is a property that is not necessary for performing behavior analysis. Therefore, the general viscosity used in the analysis must be found.

In SBM, pre-process pressure and process pressure are applied. The pre-process pressure is the air pressure applied on the stretching rod to prevent the stretching

Received (October 27, 2017), Review Result (February 15, 2018), Accepted (February 23, 2018) 
rod from completely sticking when the preform is in contact with the preform to stretch the preform, and the process pressure is the air pressure applied to secure the preform to the mold wall during or after stretching. Since it is not possible to observe how these two pressures are applied in the mold in time, appropriate interpretation is necessary. Finding this appropriate interpretation is one of the purposes of this study.

Since this whole internal process is performed in an invisible state, most of the studies have been carried out at arbitrary assumptions and show a great difference from the actual product[4]. Therefore, it is necessary to generalize the analysis method so as to obtain the same result as the actual product, so that the SBM design can be performed as efficiently as possible in the field.

\section{Theoretical Backgrounds}

\subsection{Definition and Characteristics of SBM}

SBM is generally one of the methods of molding plastic containers. After the preform is heated to a elongation proper temperature more than the softening point and less than the glass transition point, it is inserted into a mold to be formed, and then it is stretched by a stretching rod at the neck portion thereof and pre-process pressure is applied in the direction in which the stretching rod is inserted at the same time[5]. After elongation of the stretching rod has proceeded to some extent, a process pressure is applied, and the preform is brought into close contact with the wall surface of the mold to solidify it. The SBM is characterized in that the stretched state further improves the tensile strength by closely adhering the interpolymer alignment state, and improves the impact strength at the time of dropping, transparency of the product, and weight reduction[6]. Generally, a large amount of PET material is used because the non-crystalline polymer such as PET has a wide range of thermoplasticity and is therefore easier to mold than a partially crystalline PP.

\subsection{Viscosity of General Newtonian Fluid}

The elastic-viscoplastic model is known to be most effective in predicting the accuracy of SBM behavior of polymer compounds such as plastics[7]. However, the model has a disadvantage of increasing the number of material tests and the difficulty of the analysis technique[8]. In general fluid analysis, it is divided into Newtonian fluid and non-Newtonian fluid. In the case of plastic, it assumes Bingham Plastic as the non-Newtonian fluid and performs the analysis. However, this is a more appropriate method for analyzing extreme cases of plastic materials or fluids such as clay, toothpaste, and the like. Considering that the complicated method is inconvenient to use in the field, it is more appropriate to perform the analysis assuming that it is a general Newtonian fluid. For a general fluid such as water, oil, and air, the linear relationship between the applied shear and the resulting strain can be expressed as Equation (1).

$\tau \propto \frac{\delta \theta}{\delta t}$

This can be expressed as a two-dimensional geometric equation (2).

$\tan \delta \theta=\frac{\delta u \delta t}{\delta y}$ 
The equation (3) is expressed as the relationship between the shear $\operatorname{strain}(\mathrm{d} \theta / \mathrm{dt})$ and the velocity gradient(du/dy) when reaching the limit of infinitesimal change.

$\frac{d \theta}{d t}=\frac{d u}{d y}$

Therefore, from equation (1), for a general linear fluid, the applied shear stress is proportional to the velocity gradient. The proportional constant is the viscosity coefficient.

$\tau=\mu \frac{d \theta}{d t}=\mu \frac{d u}{d y}$

The equation (4) is dimensionally consistent. Therefore, the viscosity coefficient has the dimension of stress-time $[\mathrm{FT} / \mathrm{L} \wedge 2]$ or $[\mathrm{M} /(\mathrm{LT})]$. This is $\mathrm{kg} / \mathrm{ms}$ in SI units. The linear fluid following the above equation is called 'Newtonian Fluid' [9].

\section{Simulation using Finite Element Analysis}

\subsection{Only Stretch Analysis}

Figure 1 shows the sequence of the processes that occur in the nonvisual state of the SBM process.

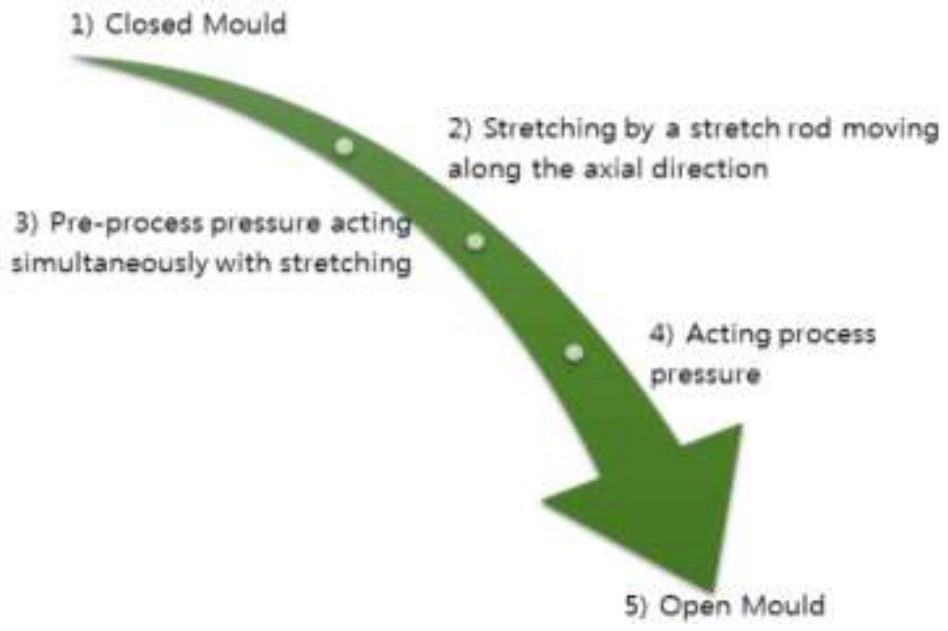

Figure 1. The Sequence of Processes that Occur in an Invisible State During the SBM Process

In this paper, we will analyze the processes of 2) 4) individually, check each optimal state, and compare the analysis thickness with the actual product thickness.

The analytical program used is Ansys PolyFlow, and fluid analysis is assumed to be a general Newtonian fluid. Since we don`t know the value of the viscosity among the physical properties of the preform, we try to find the optimum viscosity by using appropriate assumptions.

In the analysis of the molding behavior for the case of only stretch, we will try to understand the molding behavior at a high viscosity considering the viscosity of a general polymer compound.

Table 1 and Table 2 summarize the material properties and molding conditions used in the analysis of the molding behavior according to the change of viscosity in the case of Only Stretch, and Figure 2 to Figure 6 are the result of the analysis of the molding conditions. 
Table 1. Properties of Preforms for the Change of the Viscosity in Only Stretch Case

\begin{tabular}{|l|l|l|}
\hline Item & Preform & Note \\
\hline Density & $1400 \mathrm{~kg} / \mathrm{m}^{3}$ & \multirow{3}{*}{ Fixed } \\
\cline { 1 - 2 } Time & $0.1 \mathrm{~s}$ & \\
\hline Velocity & $0.25 \mathrm{~m} / \mathrm{s}$ & \\
\hline Thickness & $3 \mathrm{~mm}$ & \\
\hline
\end{tabular}

Table 2. Molding Conditions for Viscosity Change in Only Stretch Case

\begin{tabular}{|l|l|l|}
\hline Item & Viscosity $(\mathbf{n})$ & Note \\
\hline \multirow{4}{*}{ Stretch Rod } & $100,000 \mathrm{cp}$ & \\
\cline { 2 - 2 } & $300,000 \mathrm{cp}$ & \multirow{2}{*}{$\begin{array}{l}\text { High viscosity of } \\
\text { general copolymer } \\
\text { compounds }\end{array}$} \\
\cline { 2 - 2 } & $500,000 \mathrm{cp}$ & \\
\cline { 2 - 2 } & $700,000 \mathrm{cp}$ & \\
\cline { 2 - 3 } & $900,000 \mathrm{cp}$ & \\
\hline
\end{tabular}

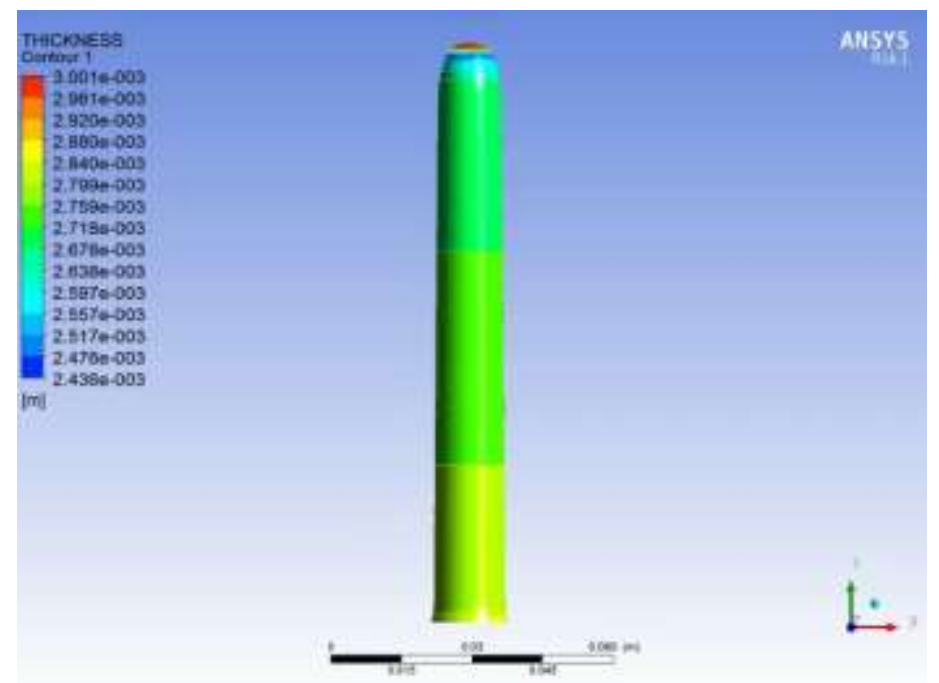

Figure 2. Only Stretch Molding Behavior at $n=100,000 \mathrm{cp}$

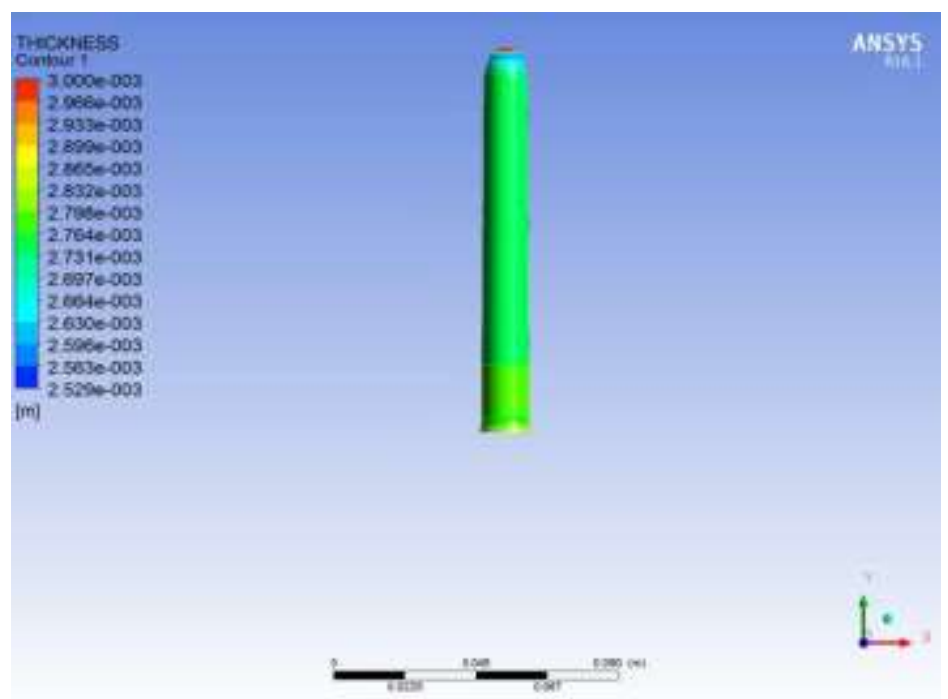

Figure 3. Only Stretch Molding Behavior at $n=300,000 \mathrm{cp}$ 


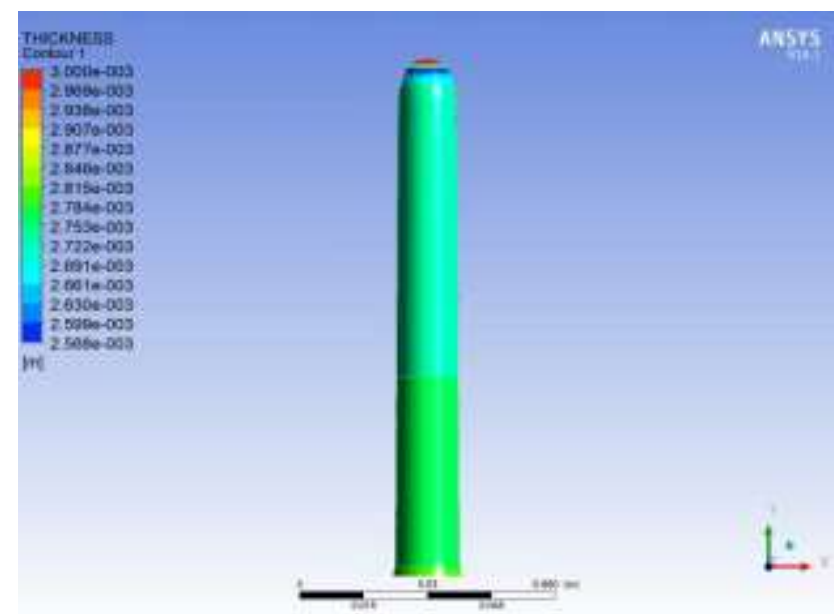

Figure 4. Only Stretch Molding Behavior at $\mathrm{n}=500,000 \mathrm{cp}$

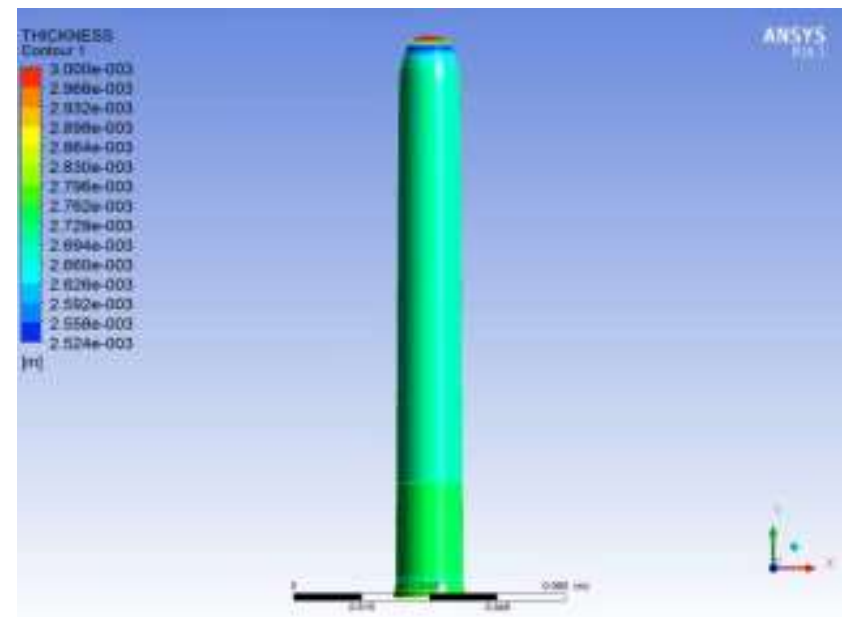

Figure 5. Only Stretch Molding Behavior at $n=700,000 \mathrm{cp}$

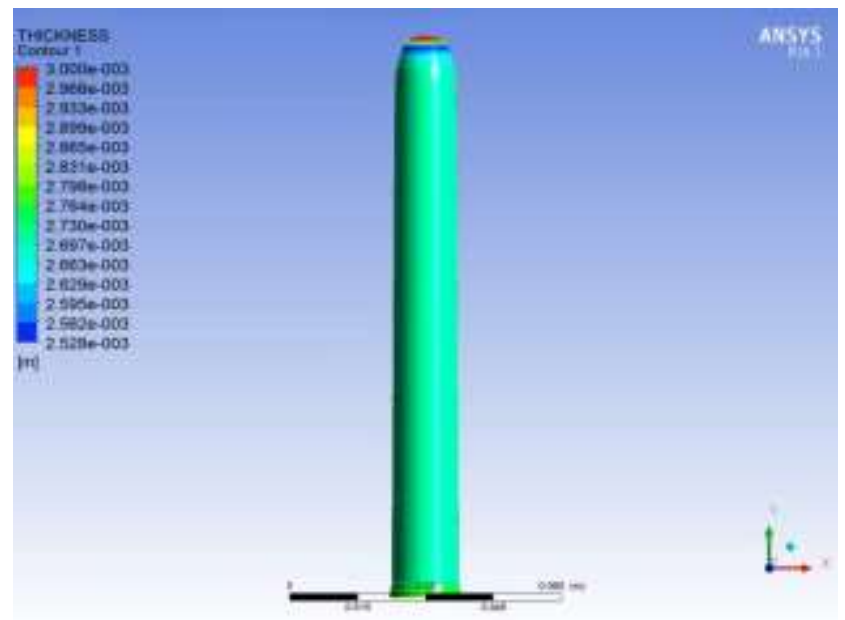

Figure 6. Only Stretch Molding Behavior at $n=900,000 \mathrm{cp}$

From the analysis results, the higher the viscosity value, the more uniform the thickness distribution on the simple stretching. However, in the case of PET, since the viscosity value is lowered when preheating before processing, the viscosity of 
the general polymer compound is $100,000 \mathrm{cp}$ or less. There is a restriction condition that interpretation should proceed.

The analysis of the viscosity of $100,000 \mathrm{cp}$ or less should be taken into account in the analysis of the only blow which will be carried out next, without further progress in the case of only stretch, since it is better to observe in the blow analysis than in the stretch analysis.

\subsection{Only Blow Analysis}

In the SBM process, the only observation of the molding behavior for the case of Only Blow is the thickness distribution on the side of the bottle rather than the overall behavior.

In the case of only blow, since the stretch process is omitted at first, the entire thickness of the bottle becomes thinner as it goes to the bottom of the bottle. Therefore, the result of the analysis obtained in this process can be focused on the molding behavior of the side of the bottle.

In the SBM analysis, time-dependent analysis is generally performed. However, for parts that do not require complex analysis, it is necessary to proceed with interpretation assuming time independent analysis. The pressure acting on the SBM interpretation generally proceeds to a time-related interpretation. As it pressurizes the preforms over time.

Table 3 and Table 4 summarize the material properties and molding conditions used in the analysis of the molding behavior according to the viscosity change in the case of only blow, Figure 7 and Figure 8 are the result of analysis for the corresponding molding conditions.

\section{Table 3. Properties of Preforms for the Change of the Viscosity in Only Blow} Case

\begin{tabular}{|l|l|l|}
\hline Item & Preform & Note \\
\hline Density & $1400 \mathrm{~kg} / \mathrm{m}^{3}$ & Fixed \\
\hline Thickness & $3 \mathrm{~mm}$ & \\
\hline
\end{tabular}

Table 4. Molding Conditions for Viscosity Change in Only Blow Case

\begin{tabular}{|l|l|l|}
\hline Item & Viscosity $(\mathbf{n})$ & Note \\
\hline \multirow{2}{*}{ Stretch Rod } & $100,000 \mathrm{cp}$ & $\begin{array}{l}\text { Viscosity of general } \\
\text { copolymer compounds }\end{array}$ \\
\cline { 2 - 3 } & $50,000 \mathrm{cp}$ &
\end{tabular}

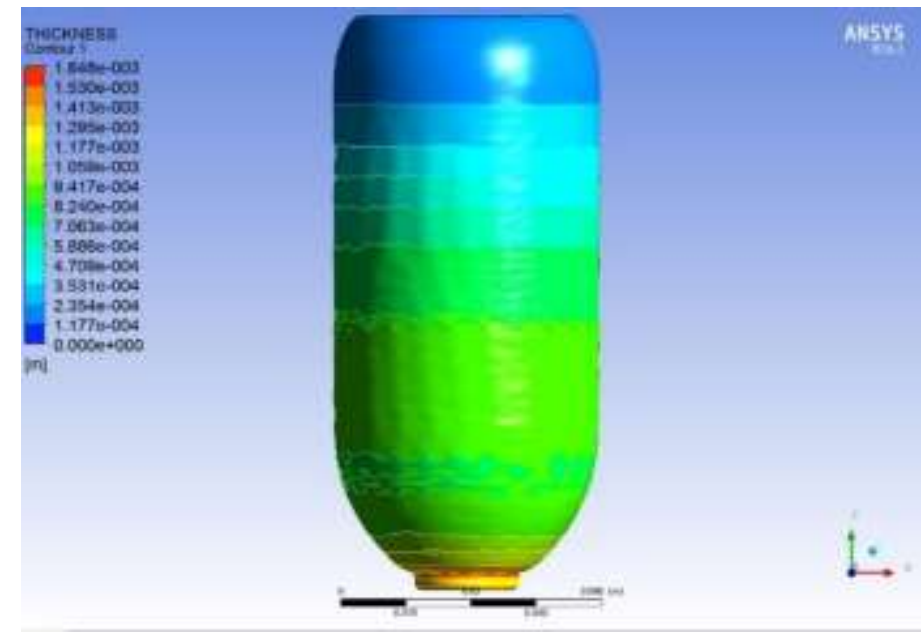

Figure 7. Molding Behavior at $n=100,000 \mathrm{cp}$ for Only Blow 


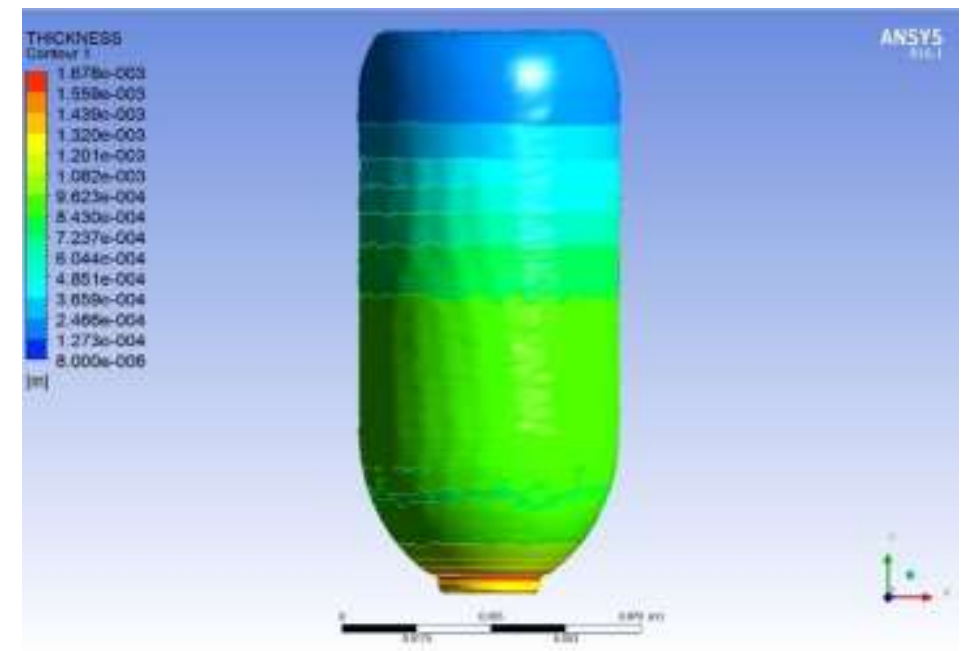

Figure 8. Molding Behavior at $n=50,000 \mathrm{cp}$ for Only Blow

In the analysis of the behavior according to the viscosity for the only blow case, the distribution of the thickness difference at the side of the bottle side is not so large in the two analyzes with the difference of 2 times. However, there is one difference between these two analysis results, which is the time it takes to blow up to that shape. In the case of Figure 7, it took 0.0038 seconds and in Figure 8, it took 0.0028 seconds. Therefore, in the case of only blow, the time taken depends on the viscosity. Generally, the lower the viscosity, the faster the mold is reached.

\subsection{Stretch Blow Analysis}

\subsubsection{Apply Ramp Function for the Pressure}

Previously, SBM analysis generally referred to time-related interpretations for the pressure. Because, in the combined analysis of a combination of stretch and blow, the process pressure acting on the preform should act at an appropriate time, resulting in a product having a uniform thickness on the side of the bottle. Therefore, the process pressure must be applied in the form of a constant function related to time. The general method used here is the Ramp function.

Figure 9 shows the general form of the Ramp function.

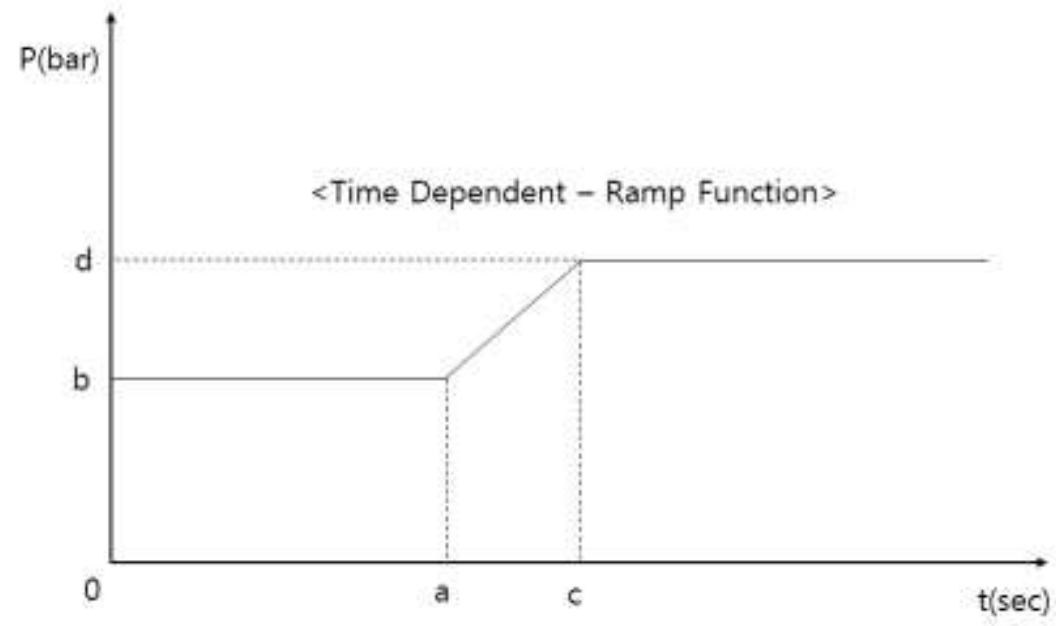

Figure 9. Graph Showing the Correlation of time to Pressure and Time 
Among the values of $\mathrm{a}, \mathrm{b}, \mathrm{c}$, and $\mathrm{d}$ for the Ramp function described later, a and $\mathrm{c}$ are time, and $b$ and $d$ are coefficients multiplying the pressure. The Ramp function application for pressure in Ansys Polyflow can be expressed as equation (5).

$P_{\text {output }}=f(t)_{\text {ramp }} \times P_{\text {input }}$

Where $P_{\text {output }}$ is the applied pressure value, $f(t)_{\text {ramp }}$ is the Ramp function, and $P_{\text {input }}$ is the pressure value entered by the user. That is, the $b$ value and the $d$ value inputted by the user are multiplied by $\mathrm{P}_{\text {input }}$ and applied to the corresponding time. Therefore, the values of $\mathrm{a}, \mathrm{b}, \mathrm{c}$, and $\mathrm{d}$ applied in the subsequent analysis are summarized in Table 5 below.

\section{Table 5. Variables of Ramp Function for Blow Input in Ansys Polyflow}

\begin{tabular}{|l|l|l|l|l|}
\hline \multirow{2}{*}{ To Pressure } & \multicolumn{4}{|l|}{ Ramp Function Variable } \\
\cline { 2 - 5 } & $\mathrm{a}$ (second) & $\mathrm{b}$ & $\mathrm{c}$ (second) & $\mathrm{d}$ \\
\hline Variable Value & 0.1 & 0 & 0.101 & 1 \\
\hline
\end{tabular}

There is an interval of 0.001 second between the a and c values. In this study, the pressure applied to this interval was assumed to be the pre-process pressure. For further analysis, additional analysis and data accumulation will be needed for the influence of the pre-process pressure.

\subsubsection{Apply Ramp Function for the Velocity of the Stretch Rod}

The stretch rod will performs the analysis with a process time of 0.1 second, as in the case of Only Stretch. Since the stretch rod will moves by a total displacement of $25 \mathrm{~mm}$ inside the mold, this will also be applied to the analysis using the Ramp Function. Table 6 shows the set values of the Ramp Function for the speed of the stretch rod.

Table 6. Variables of Ramp Function for Stretch Input in Ansys Polyflow

\begin{tabular}{|l|l|l|l|l|}
\hline \multirow{2}{*}{ To Stretch Rod } & \multicolumn{4}{|c|}{ Ramp Function Variable } \\
\cline { 2 - 5 } & a(second) & b & c(second) & d \\
\hline Variable Value & 0.1 & 1 & 0.101 & 0 \\
\hline
\end{tabular}

Stretch Blow Molding composite analysis is performed with the applied values as shown in Table 6.

\subsubsection{Composite Analysis of Stretch Rod Applied to the Ramp Function}

Only Blow analysis confirmed that the preform reached the mold in a very short time, so we set the ramp function so that the process pressure is applied after $0.1 \mathrm{~s}$ after the completion of Only Stretch. The analysis conditions and analysis results of the SBM analysis under the condition of Table 5 and Table 6 are shown in Figure 10 and Figure 11. As shown in the figure, it can be seen that both have similar side wall thickness distributions. However, the most similar distribution to the thickness of the bottle side is the result of interpreting the viscosity at 50,000 cp. Also, it can be seen that the lower the viscosity, the faster the contact time of the molded product with the preform is. The difference in total contact time from 100,000 cp to 50,000 $\mathrm{cp}$ is not much different from $0.000014 \mathrm{~s}$. However, considering the shortening of the process time, there is a need for further analysis with more viscosity. 


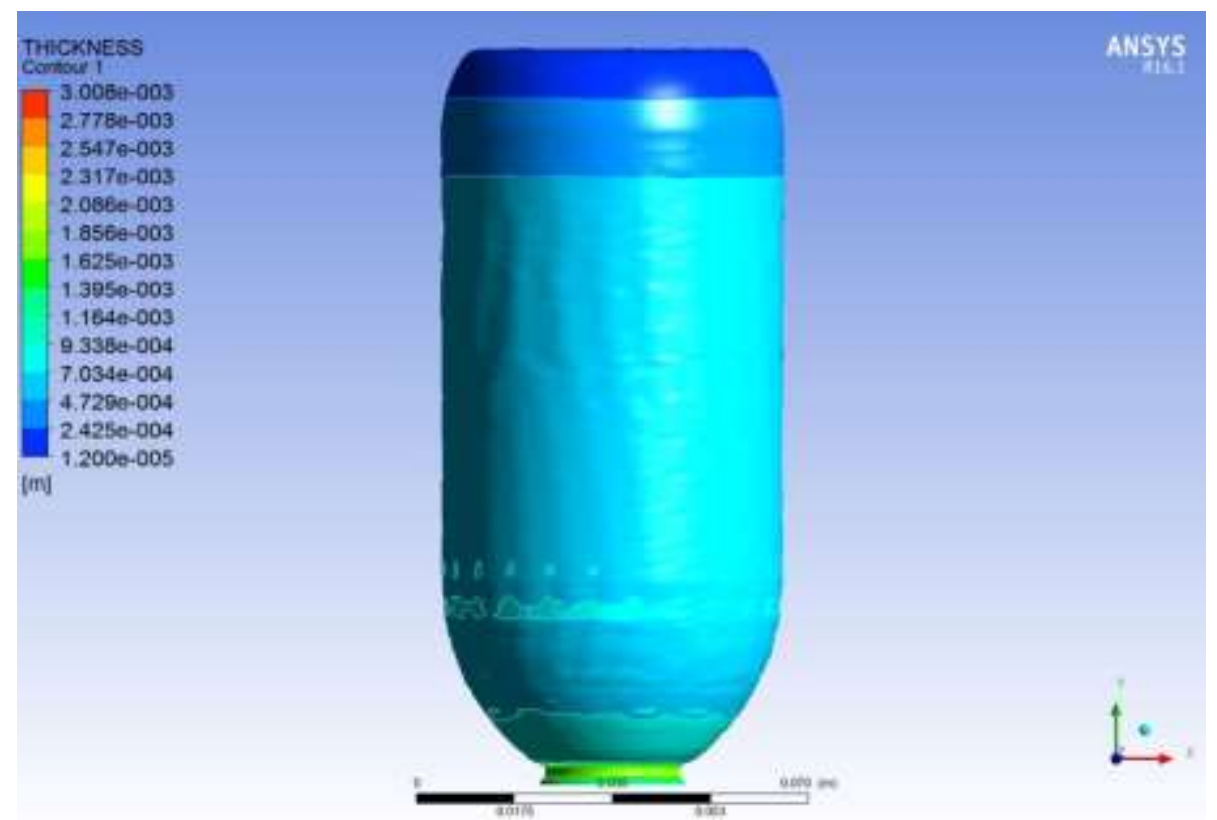

Figure 10. SBM Analysis Results at $n=100,000 \mathrm{cp}$ and $t=0.101364 \mathrm{~s}$

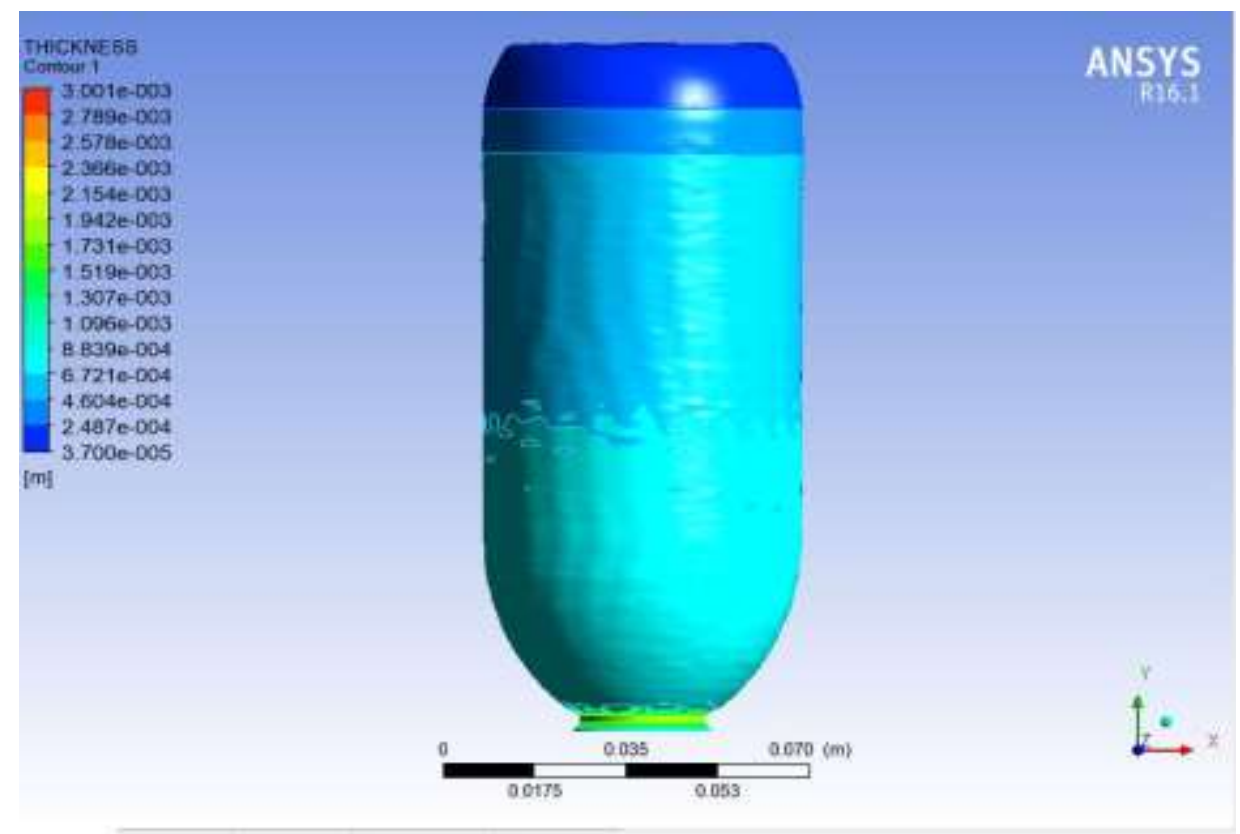

Figure 11. SBM Analysis Results at $n=50,000 \mathrm{cp}$ and $t=0.101350 \mathrm{~s}$

\subsection{Comparison of Thickness between Actual Product and Analysis Result}

The SBM interpretation is based on assumptions estimated to be appropriate and should be compared with the actual product to ensure the validity of the interpretation. Therefore, the products actually produced were dimensioned at $5 \mathrm{~mm}$ intervals with a micrometer. Figure 12 shows the actual product, Table 7 is the table comparing the actual product with the analysis result. 


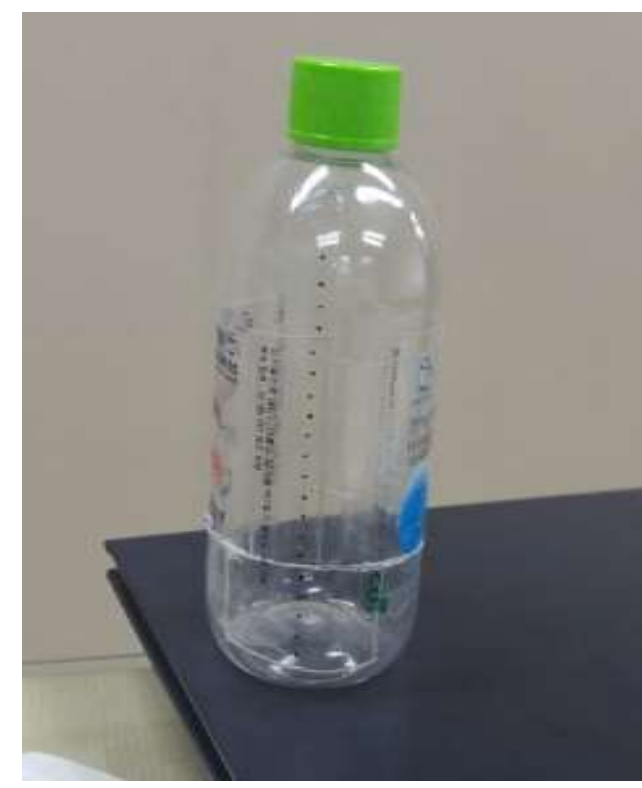

Figure 12. Actual Product and Thickness Measurement Location Corresponding to SBM Analysis

Table 7. Comparison of Lateral Thickness of Actual Product with $n=50,000$ cp SBM Analysis Result

\begin{tabular}{|c|c|c|c|}
\hline $\begin{array}{l}\text { Distance from a } \\
\text { bottom side( }(\mathrm{mm})\end{array}$ & $\begin{array}{c}\text { Thickness of real } \\
\text { bottle }(\mathrm{mm})\end{array}$ & $\begin{array}{c}\text { Thickness of Analysis } \\
\text { bottle( }(\mathrm{mm})\end{array}$ & Note \\
\hline 0 & \multicolumn{2}{|c|}{ Bottom side of a bottle } & \\
\hline 10 & 0.55 & \multirow{19}{*}{$0.67 \sim 0.46$} & \\
\hline 15 & 0.54 & & \\
\hline 20 & 0.54 & & \\
\hline 25 & 0.53 & & \\
\hline 30 & 0.52 & & \\
\hline 35 & 0.50 & & \\
\hline 40 & 0.49 & & \\
\hline 45 & 0.48 & & \\
\hline 50 & 0.45 & & \\
\hline 55 & 0.41 & & \\
\hline 60 & 0.38 & & \\
\hline 65 & 0.37 & & \\
\hline 70 & 0.37 & & \\
\hline 75 & 0.37 & & \\
\hline 80 & 0.35 & & \\
\hline 85 & 0.35 & & \\
\hline 90 & 0.37 & & \\
\hline 95 & 0.44 & & \\
\hline 100 & 0.52 & & \\
\hline
\end{tabular}

Table 7 shows that the actual bottle thickness has a thickness distribution of 0.6 to $0.4 \mathrm{~mm}$ from the bottle bottom, while the analytical result shows a distribution of 0.67 to $0.46 \mathrm{~mm}$. The analysis result of Ansys Polyflow is visible only as above, and detailed data for each node can not be confirmed. Since the analysis result is 50,000 
$\mathrm{cp}$, it can be confirmed that more accurate analysis results can be obtained by performing the analysis with a lower value of $\mathrm{cp}$.

\section{Conclusion}

In this study, the behavior of PET bottles using SBM process and the FEM analysis to predict the micro change of film label fusion were performed. In particular, since the SBM process itself proceeds in an invisible state that can not be seen from the outside during the process, the analysis is repeated with appropriate assumptions, and the property values and the analysis process are modified so that results similar to the actual dimensions are obtained. As a result, the following conclusions were obtained.

1) In the analysis of the only stretch case, it was confirmed that a PET bottle having a more uniform thickness was formed as the operation time of the stretch process became longer. In the case of the viscosity, a PET bottle with a more uniform thickness could be formed as the viscosity increased. However, if the process time is too long, it will have a direct adverse effect on the production amount, so it can be confirmed that it should be limited to about 0.1 second.

2) In the case of only blowing, it was confirmed that the molding process could be completed in a very short time. Also, it was confirmed that the lower the viscosity value, the smaller the time taken for the PET bottle to completely contact the mold. Therefore, it was confirmed that the optimum value should be found at $50,000 \mathrm{cp}$ or less among the values of $100,000 \mathrm{cp}$ or less, which is the general viscosity of the polymer compound.

3) In the case of Stretch Blow case, it was confirmed that the thickness of the side of the bottle was ideally formed when the process pressure was applied before and after the end of the stretch process. However, we could confirm that the bottom part of the bottle is different from the actual product thickness.

4) When the bottle side thicknesses of the actual PET bottle and the analyzed PET bottle are compared, it is confirmed that the thickness of the actual molded article is thinner. It is confirmed that the uniform distribution of the side wall thickness is similar to that of the bottle side, which is expected to be solved by performing more repeated analysis by changing the viscosity value.

Finally, Considering the amount of micro change in film fusion, the label film is attached to the side of the bottle, so that the pressure is applied in a direction perpendicular to the surface in a very short time. After the molded article contacts the mold, it is expected that micro change behaviors will occur from the neck to the bottom of the bottle when the overall behavior of the label film is observed. Even if a 3D molding such as a groove is added to the mold, the micro change behavior along the above is expected to occur.

\section{Acknowledgments}

This paper is a revised and expanded version of a paper entitled "Analysis of Molding of PET Bottle for 3D In-label Fusion Technology” presented at GST 2017, Jeju, Korea, December 1, 2017. 


\section{References}

[1] O. R. Kim, J. D. Kim, S. Y. Lee, Y. G. Kim, H. P. Park, C. K. Woo, H. R. Kim and C. O. Kwon, “A Numerical Study on Injection-Stretch Blow Molding For Preform Optimization", Journal of Korean Society for Precision Engineering Spring Conference, vol. 2007, no. 2, (2007) pp. 247-248.

[2] J. S. Kim and J. D. Kim, "Numerical study on the effect of the PET bottle thickness difference for blow molding process conditions", Journal of Korean Institute of Industrial Educations, vol. 34, no. 2, (2009) pp. 321-330.

[3] J. S. Kim, J. D. Kim, O. R. Kim and C. O. Kwon, "Study on numerical analysis and experiment of the injection blow molding of a preform of PET Bottle", Journal of Korea Academia-Industrial cooperation Society, vol. 9, no. 5, (2008) pp. 1119-1124.

[4] J. W. Kim, S. T. Hong, C. S. Oh and M. Y. Lyu, "Blowing Characteristics of PET Bottle for Blowing Conditions", Journal of The Korean Society For Technology of Plasticity Autumn Conference, vol. 2002, no. $10, \mathbf{( 2 0 0 2 )}$ pp. $147-150$.

[5] M. J. Song, H. K. Kim, H. P. Park and B. S. Cha, "Viscoplastic FEM Analysis of Stretch Blow Molding Process using Tensile Test Result", Journal of The Korean Society For Technology of Plasticity Spring Conference, vol. 2013, no. 5, (2013) pp. 285-287.

[6] S. T. Joo, Y. H. Kim and M. Y. Lyu, "Analysis of Forming Processes of PET Bottle using a Finite Element Method", Journal of Transactions of Materials Processing, vol. 10, no. 6, (2001) pp. 525-533.

[7] H. K. Kim, B. S. Cha, H. P. Park and Y. S. Kim, "Viscoplastic FEM Analysis of Stretch Blow Molding Process", Journal of Korean Society for Precision Engineering Autumn Conference, vol. 2010, no. 11, (2010) pp. 833-834.

[8] H. K. Kim, H. P. Park and B. S. Cha, "A Simulation of Injection Stretch-Blow Molding Process by Using A Viscoplastic Model”, Journal of Mold Processing Symposium, vol. 2010, no. 11, (2010) pp. 107-110.

[9] Frank M. White, "Fluid Mechanics", Korea McGraw-Hill, Seoul, vol. 1, (2013) pp. 26-28.

[10] Frank M. White, "Fluid Mechanics", Korea McGraw-Hill, Seoul, vol. 1, (2013) p. 49.

\section{Authors}

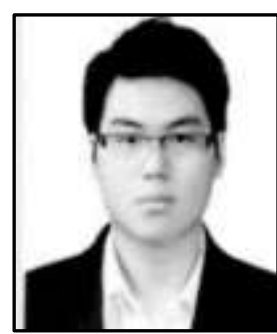

Seong-Gyu Cho, he received B.Sc degree in Mechanical System Engineering from Kyonggi University, Suwon, Korea, in 2016. He is currently in the Mater's course of Mechanical Engineering in Kyonggi University. He is interested in 3D printing and numerical simulation such as the structural analysis.

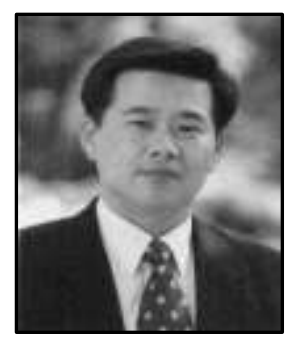

Joon-Seong Lee, he received B. Sc (1986) and M.Sc (1988) degrees in Mechanical Eng. from SungKyunKwan University, Suwon, Korea. He worked as a professor during 1988-1991 in the Dept. of Mechanical Eng. of Korea Military Academy. He received $\mathrm{Ph} . \mathrm{D}$. degree in engineering from the University of Tokyo in 1995. Now he is a professor in the Dept. of Mechanical System Engineering of Kyonggi University. His current research interest is the development of an intelligent simulation system that will effectively combine numerical simulations such as the finite element method and soft computing techniques, and its application to various artifacts such as micromachines. 